\title{
Perfil Epidemiológico de Pacientes Diagnosticados com Carcinoma Epidermoide Oral em Passo Fundo, Brasil
}

doi: https://doi.org/10.32635/2176-9745.RBC.2021v67n4.1397

\author{
Epidemiological Profile of Patients Diagnosed with Oral Squamous Cell Carcinoma in Passo Fundo, Brazil \\ Perfil Epidemiológico de Pacientes Diagnosticados con Carcinoma Epidermoide Oral en Passo Fundo, Brasil
}

Luana Soares Kuze'; Gisele Rovani²; Adriano Pasqualotti ${ }^{3}$; Ferdinando De Conto ${ }^{4}$; Mateus Ericson Flores ${ }^{5}$; Thales Henrique Jincziwski Ponciano $^{6}$

\section{RESUMO}

Introduçáo: O carcinoma epidermoide é uma patologia que corresponde a $90 \%$ dos tumores malignos da cavidade oral. Objetivo: Avaliar o perfil epidemiológico, o tratamento e a sobrevida dos pacientes diagnosticados com carcinoma epidermoide oral em um hospital referência na Regiāo Norte do Estado do Rio Grande do Sul, Brasil. Método: Estudo observacional retrospectivo, com análise dos prontuários dos pacientes com diagnóstico dessa patologia, no período de 1993 a 2008, no banco de dados desse hospital. Os dados foram analisados pelo programa estatístico SPSS versão 18 utilizando testes paramétricos e não paramétricos. Resultados: Foram coletados e analisados dados de 303 prontuários que revelaram que 88,4\% eram homens, leucodermas, com maior incidência na faixa etária entre 51 e 60 anos. A associação de álcool e tabaco foi observada em $84,7 \%$ dos pacientes. O local mais afetado foi a língua (45,5\%) e a maioria dos casos apresentou estádios tardios III e IV com uma sobrevida de 17,83 meses. Embora o tratamento cirúrgico tenha sido o mais realizado $(55,9 \%)$ e apresentado a maior sobrevida, náo foi observada significância estatística deste com relaçáo aos demais tratamentos ( $\mathrm{p}=0,185)$. Conclusáo: O perfil epidemiológico dos pacientes é muito semelhante aos registros de vários países. Neste caso, o diagnóstico tardio diminuiu significativamente a expectativa de vida dos pacientes $(2,5 \mathrm{x})$, e o tratamento executado bem como o local inicial da lesão não tiveram influência significativa na sobrevida.

Palavras-chave: Neoplasias Bucais; Taxa de Sobrevida; Perfil de Saúde; Carcinoma de Células Escamosas; Prognóstico.

\section{ABSTRACT}

Introduction: The squamous cell carcinoma is a pathology accounting for $90 \%$ of all the malignant tumors found in the oral cavity. Objective: This study aimed to assess the epidemiological profile, treatment, and survival of patients diagnosed with oral squamous cell carcinoma at a reference hospital of the northern region of the state of Rio Grande do Sul, Brazil. Method: Retrospective observational study with analysis of the patients' charts diagnosed with this condition from 1993 to 2008 at this hospital database. The data collected were analyzed using the statistical program SPSS version 18 using parametric and non-parametric tests. Results: From the 303 charts analyzed, $88.4 \%$ were men, Caucasian, leukoderma, with higher incidence in the age range between 51 to 60 years old. The association of alcohol with tobacco was detected in $84.7 \%$ of the patients. The most affected site was the tongue ( $45.5 \%)$, and most of the cases were diagnosed in late stage III and IV, with survival of 17.83 months. Although surgical treatment has been performed more frequently $(55.9 \%)$ and presented higher survival rates, its statistical significance in comparison with other treatments was not demonstrated $(\mathrm{p}=0.185)$. Conclusion: The epidemiological profile of patients is very similar to the charts of several countries. In the present case, the late diagnosis significantly decreased life expectancy of patients (2.5-fold), and that the treatment, and the initial site of the lesion had no significant influence on survival.

Key words: Mouth Neoplasms; Survival Rate; Health Profile; Carcinoma, Squamous Cell; Prognosis.

\section{RESUMÉN}

Introducción: El carcinoma de células escamosas es una patología que corresponde aproximadamente a $90 \%$ de los tumores malignos de la cavidad bucal. Objetivo: Evaluar el perfil epidemiológico, el tratamiento y la sobrevida de los pacientes diagnosticados con carcinoma epidermoide oral en un hospital referencia en la región norte del estado de Rio Grande do Sul, Brasil. Método: Estudio observacional retrospectivo con los prontuarios de los pacientes con el diagnóstico de esa patología en el período de 1993 a 2008 , en el banco de datos de este hospital usando el programa estadístico SPSS versión 18 usando pruebas paramétricas y no paramétricas Resultados: Se recogieron y analizaron datos de 303 prontuarios, que revelaron que $88,4 \%$ eran hombres, leucodermas, entre 51 a 60 ańos. La asociación de alcohol y tabaco fue observada en el $84,7 \%$ de los pacientes. El local más afectado fue la lengua $(45,5 \%)$ y la mayoría de los casos ha presentado estadios tardíos III y IV con una sobrevida de 17,83 meses. Aunque el tratamiento quirúrgico fue el más realizado $(55,9 \%)$ y presentado la mayor sobrevida, no se observó la significancia estadística de éste con relación a los demás tratamientos $(\mathrm{p}=0,185)$. Conclusión: El perfil epidemiológico de los pacientes es muy similar a los registros de varios países. En este caso, el diagnóstico tardío disminuyó significativamente la expectativa de vida de los pacientes $(2,5 \mathrm{x})$ y el tratamiento realizado, así como el lugar inicial de la lesión no tuvieron influencia significativa en la sobrevida.

Palabras clave: Neoplasias de la Boca; Tasa de Supervivencia; Perfil de Salud; Carcinoma de Células Escamosas; Pronóstico.

${ }^{1}$ Centro Universitário UniFacvest. Lages (SC), Brasil. E-mail: mestreluana@hotmail.com. Orcid iD: https://orcid.org/0000-0003-4566-3142 2,4,5,6Universidade de Passo Fundo (UPF), Faculdade de Odontologia. Passo Fundo (RS), Brasil. E-mails: giselerovani@upf.br; ferdi@upf.br; mef@upf.br; thalesjincziwski@gmail.com. Orcid iD: https://orcid.org/0000-0002-4291-5749; Orcid iD: https://orcid.org/0000-0002-8077-3550; Orcid iD: https://orcid. org/0000-0002-4643-4328; Orcid iD: https://orcid.org/0000-0002-3699-4225

${ }^{3}$ UPF, Instituto de Ciências Exatas e Geociências. Passo Fundo (RS), Brasil. E-mail: pasqualotti@upf.br. Orcid iD: https://orcid.org/0000-0001-7544-9425

Endereço para correspondência: Thales Henrique Jincziwski Ponciano. Faculdade de Odontologia. Campus I - Km 171 - BR 285 - São José. Passo Fundo (RS), Brasil. CEP 99001-9470. Caixa Postal 611.E-mail: thalesjincziwski@gmail.com 


\section{INTRODUÇÃO}

Aproximadamente $10 \%$ dos tumores malignos que ocorrem no corpo humano estáo localizados na boca, sendo este o sexto tipo de câncer mais incidente no mundo. Excluindo-se o câncer de pele, o câncer bucal pode ser considerado o mais comum da regiáo de cabeça e pescoço. O carcinoma epidermoide é uma patologia que corresponde a $90 \%$ dos tumores malignos da cavidade oral ${ }^{1-3}$.

A partir da constatação de que o câncer bucal é um problema de saúde pública, é importante o conhecimento de sua magnitude no Brasil, no tocante à distribuição geográfica com estratificaçóes por idade e gênero, como base de apoio ao seu controle. A divulgação de dados estatísticos é necessária tanto para o conhecimento do quadro nacional como para estimular os profissionais da área da saúde a atuarem na prevenção e diagnóstico do câncer bucal ${ }^{1-5}$.

Levando-se em conta o crescimento e o envelhecimento da população, assim como a industrializaçáo e a urbanização, agravados pela cobertura inadequada dos serviços de saúde, é evidente a necessidade de mudar a estratégia de controle das doenças não transmissíveis, combinando açôes preventivas de promoçáo e proteção à saúde e medidas diagnósticas, especialmente aquelas de diagnóstico precoce e terapêuticas ${ }^{6,7}$. Esta pesquisa tem como objetivo avaliar o perfil epidemiológico, a forma de tratamento e sua relação de sobrevida de pacientes que foram acometidos por carcinoma epidermoide oral e submetidos ao tratamento oncológico no Hospital São Vicente de Paulo (HSVP) de Passo Fundo, RS, Brasil, no período de 1993 a 2008.

\section{MÉTODO}

Esta pesquisa foi inicialmente aprovada pelo Comitê de Ética em Pesquisa da Universidade de Passo Fundo sob número 302/2011. As informaçōes foram buscadas junto ao banco de dados do Setor de Oncologia do HSVP de Passo Fundo, RS, no período de 1993 a 2008, e tabuladas em planilha eletrônica.

Algumas informaçóes foram prioritárias a outras, como: sexo (separado em masculino e feminino), idade (agrupadas por década de vida), etnia (divididos em leucoderma e não leucoderma), estadiamento do tumor, seguimento, tipo de tratamento, entre outras; porém, em alguns registros, essas informaçóes eram incompletas. Dos 327 pacientes contidos na planilha do banco de dados, 203 não tinham dados do seguimento, ou seja, não se sabia o estado atual desse paciente.

Para fins estatísticos, o estadiamento foi agrupado em I e II; III e IV (A, B e C). Consideraram-se os locais: língua, soalho de boca, palato e outros locais não especificados. Tabagismo e consumo de álcool foram determinados como hábitos e inseridos na análise estatística, e o nível de instruçáo educacional dividido conforme as classes de grau oferecidas no Sistema Nacional de Educação.

Dados como histórico familiar, já ter recebido diagnóstico de câncer anteriormente, tipo de tratamento recebido, tempo de sobrevida e evoluçáo para óbito ou não também foram registrados e utilizados para a análise estatística. Nos cruzamentos, as variáveis que não possuíam informaçóes ou classificação foram descartadas para fins estatísticos, bem como eventuais agrupamentos se fizeram necessários para viabilizar a análise conforme descrição nos resultados.

$\mathrm{Na}$ busca por informaçóes que suprissem a necessidade da atualização dos dados, iniciaram-se contatos com os pacientes por telefone. Quando esse contato não foi possível, buscaram-se informaçôes com a Secretaria Estadual de Saúde do Estado do Rio Grande do Sul e o Sistema de Informação sobre Mortalidade (SIM) que contém dados a partir do ano de 1996 até 2009. O restante dos dados foi obtido junto às Secretarias Municipais de Saúde dos municípios, relatados nos prontuários dos pacientes no ato da internação; e, caso essa informação não fosse conseguida, o contato final era feito com os cartórios de registro civil dos referidos municípios.

Dos 327 pacientes existentes no banco de dados do HSVP, 24 foram excluídos da análise de dados desta pesquisa por razóes diversas: não se obteve informação alguma de 20 pacientes; um paciente faleceu em outro Estado brasileiro em decorrência do câncer, mas não foi obtida informação da data; um paciente estava com registro duplicado; um cometeu suicídio e outro faleceu vítima de homicídio.

Os dados coletados foram analisados pelo programa estatístico SPSS versão 18, utilizando testes paramétricos (correlação de Pearson; Kolmogorov-Smirnov) e não paramétricos (Kruskal-Wallis; Mann-Whitney; Fischer). Também foi realizada a análise de associação entre os dados de estadiamento e o tipo de tratamento e da sobrevida com outras variáveis.

\section{RESULTADOS}

Dos 303 pacientes incluídos na análise dos dados desta pesquisa, $88,4 \%(n=268)$ eram homens e apenas $11,6 \%(\mathrm{n}=35)$, mulheres, obtendo-se uma proporção de 1:7,3 (M:H).

A faixa etária mais acometida pela patologia estudada foi de 51 a 60 anos de idade com $37 \%$ dos casos $(n=111)$, seguida pela faixa de 61 a 70 anos, representando $25 \%$ da amostra $(n=75)$. A idade média dos pacientes acometidos 
pelo carcinoma foi de 58,18 anos, com um intervalo de 30 a 85 anos.

O grau de instruçáo dos pacientes foi analisado. Dos $78,5 \%(n=238)$ que informaram esse dado, foi observada a seguinte distribuição: Ensino Fundamental 78,6\% $(\mathrm{n}=187)$, Ensino Médio 10,9\% ( $\mathrm{n}=26)$, analfabetos 5,9\% $(\mathrm{n}=14)$, e Superior $4,6 \%(\mathrm{n}=11)$.

Os dados de etnia foram ajustados para fins estatísticos e considerados da seguinte forma: leucodermas $93,4 \%$ $(\mathrm{n}=283)$ e não leucodermas (melanodermas, faiodermas e xantodermas) com $6,6 \%(n=20)$.

Dos pacientes que informaram o histórico familiar de câncer $(51,5 \% ; n=156), 38,5 \%(n=60)$ relataram casos na família, contra $61,5 \%(n=96)$ que não apresentaram.

Os pacientes que não receberam diagnóstico de câncer anteriormente somaram $69,3 \%(\mathrm{n}=210)$, já $22,8 \%$ dos pacientes $(n=69)$ receberam esse diagnóstico, porém náo realizaram tratamento e $7,9 \%(\mathrm{n}=24)$ diagnóstico e tratamento anteriormente.

O tabagismo foi informado em 74,6\% ( $\mathrm{n}=226)$ dos prontuários e esteve presente nos hábitos de 90,7\% $(\mathrm{n}=205)$ dos pacientes. $\mathrm{O}$ consumo de álcool foi relatado por $69,0 \%(\mathrm{n}=209)$ dos pacientes, destes, $87,1 \%(\mathrm{n}=182)$ eram etilistas. A associação de tabaco e álcool foi observada em $84,7 \%(n=177)$ pacientes.

A localização topográfica da lesão inicial foi agrupada e considerados os seguintes locais: língua 45,5\% ( $\mathrm{n}=138)$, outros locais não especificados $23,8 \%(\mathrm{n}=72)$, palato $13,2 \%(\mathrm{n}=40)$, assoalho da boca $11,9 \%(\mathrm{n}=36)$, lábio $4,3 \%(n=13)$, gengiva $1,3 \%(n=4)$.

O estadiamento do tumor no momento do diagnóstico foi observado em $65 \%$ dos prontuários $(n=197)$. O diagnóstico com estadiamentos avançados III e IV foi observado na grande maioria dos casos, 77,2\% ( $\mathrm{n}=152)$ contra 22,8\% $(\mathrm{n}=45)$ dos diagnósticos com estadiamentos iniciais I e II. Na Tabela 1, observa-se a distribuição completa dos casos.

O tratamento utilizado em cada caso foi descrito em 299 prontuários e mostrou-se distribuído em procedimento cirúrgico $(55,9 \%, \mathrm{n}=167)$, radioterapia $(6,0 \%, \mathrm{n}=18)$ e quimioterapia $(4,7 \%, \mathrm{n}=14)$; a combinação desses tipos de tratamentos foi registrada em 19,4\% ( $n=58)$ dos casos, nenhum tratamento ocorreu em 14,0\% $(n=42)$ dos pacientes.

Com relação ao seguimento, observou-se que $68,6 \%$ dos pacientes $(\mathrm{n}=208)$ foram a óbito e 31,4\% ( $\mathrm{n}=95)$ deles estavam vivos. Dos 208 pacientes que foram a óbito, a média de sobrevida foi de 25,75 meses, com um intervalo de -10,6 e 149,1 meses (Gráfico 1).

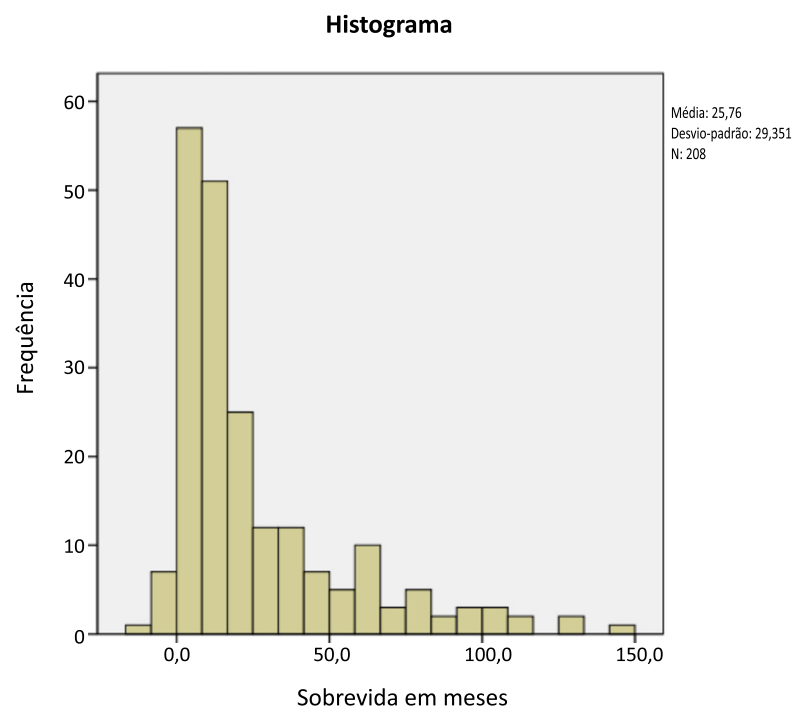

Gráfico 1. Frequência da sobrevida global dos pacientes que foram a óbito em decorrência do câncer, período de 1993 a 2008. Passo Fundo, RS. Brasil

Para fins estatísticos, foram agrupadas as formas de tratamento radioterapia e quimioterapia em razão de o número de casos para ambos ser pequeno; para os estadiamentos I e II, observou-se que o uso do tratamento cirúrgico foi significativo $(\mathrm{p}<0,001)$; já para o estadiamento IV, foi o uso de terapias combinadas. Os tratamentos que envolviam radioterapia e quimioterapia não foram significativos para nenhum tipo de estadiamento.

A remoção cirúrgica do tumor foi realizada em 95,5\% ( $\mathrm{n}=43)$ dos pacientes em estádios I e II. Radioterapia ou quimioterapia em $2,2 \%(\mathrm{n}=1)$ e tratamentos combinados em 2,2\% ( $n=1)$. Já em estádios avançados da doença, III e

Tabela 1. Distribuição completa dos casos por estadiamento, sexo, óbitos e sobrevida, período de 1993 a 2008, Passo Fundo, RS. Brasil

\begin{tabular}{cccccccccc}
\hline Estádio & \multicolumn{2}{c}{ Masculino } & \multicolumn{2}{c}{ Feminino } & \multicolumn{2}{c}{ Total } & \multicolumn{2}{c}{ Óbito } & $\begin{array}{c}\text { Sobrevida em } \\
\text { meses }\end{array}$ \\
\hline & $\mathbf{n}$ & $\%$ & $\mathbf{n}$ & $\%$ & $\mathbf{n}$ & $\%$ & $\mathbf{n}$ & $\%$ & 36,5 \\
I & 19 & $10,7 \%$ & 6 & $31,6 \%$ & 25 & $12,7 \%$ & 9 & $36,0 \%$ & 53,6 \\
II & 19 & $10,7 \%$ & 1 & $5,3 \%$ & 20 & $10,2 \%$ & 12 & $60,0 \%$ & 22,8 \\
IV & 31 & $17,4 \%$ & 5 & $26,3 \%$ & 36 & $18,3 \%$ & 24 & $66,7 \%$ & 16,6 \\
TOTAL & 109 & $61,2 \%$ & 7 & $36,8 \%$ & 116 & $58,9 \%$ & 91 & $78,4 \%$ & \\
\hline
\end{tabular}


IV, o tratamento cirúrgico foi realizado em 40,1\% (n=61) dos pacientes, a radioterapia ou quimioterapia em 15,1\% $(\mathrm{n}=23)$ destes, e a combinação de dois ou mais tratamentos em $34,2 \%(n=52)$ dos pacientes.

Utilizando-se a correlaçáo de Pearson, observou-se que, em estádios III e IV, a ocorrência na língua foi maior do que nos demais sítios, porém não foi observada diferença estatística $(\mathrm{p}=0,073)$.

Também não houve significância estatística $(\mathrm{p}=0,197)$ entre tratamento e seguimento por meio da correlação de Pearson. Sendo que o tratamento utilizado não influenciou significativamente no seu seguimento. Isso se deu também entre local da lesão e seguimento $(\mathrm{p}=0,159)$.

Quando observado o estadiamento em relação ao seguimento por intermédio do teste de Fischer, verificou-se que, nos estádios I e II, encontrava-se o maior número de pacientes vivos 39,34\% ( $\mathrm{n}=24)$; já nos estádios III e IV, maior número de mortos $84,56 \%(\mathrm{n}=115)$, havendo diferença estatística entre os grupos $(\mathrm{p}<0,001)$. O risco relativo do paciente diagnosticado em estádios avançados III e IV aumentou 2,5 vezes a chance de ir a óbito do que quando diagnosticado precocemente nos estádios I e II.

Dos 136 pacientes mortos e que tinham informação do estadiamento, foi realizado o cálculo da sobrevida (Gráfico 2). Nos estádios I e II, encontrou-se sobrevida de 46,25 meses; nos estadiamentos III e IV, de 17,83 meses. Pelo teste de Mann-Whitney, observa-se que a diferença foi significativa $(\mathrm{p}=0,003)$.

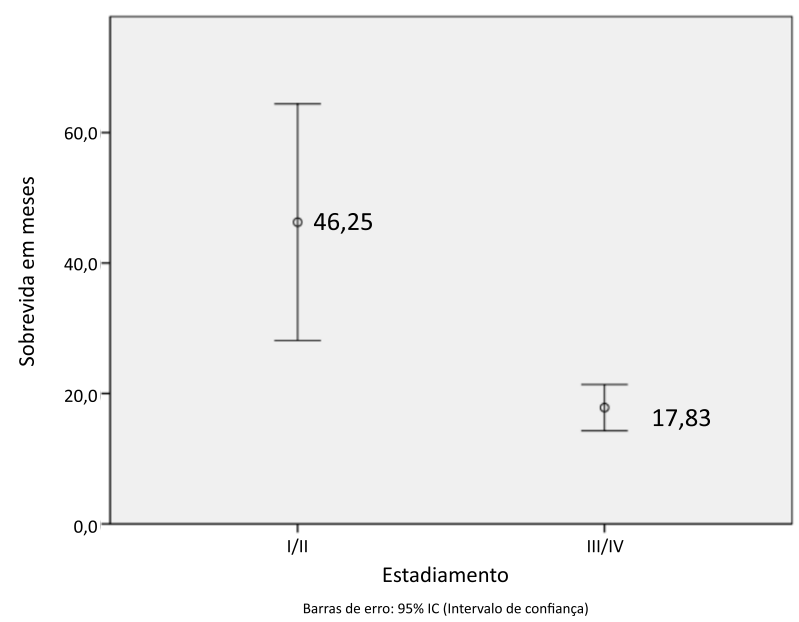

Gráfico 2. Intervalo e média da sobrevida dos pacientes que foram a óbito, segundo o estadiamento, período de 1993 a 2008. Passo Fundo, RS, Brasil

Dos 170 pacientes que receberam tratamento e foram a óbito, a sobrevida após o tratamento cirúrgico foi em média de 33,19 meses. No tratamento com radioterapia ou quimioterapia, a média de sobrevida foi de 21,13 meses. Quando houve uma combinação de dois ou mais tratamentos, a sobrevida foi de 15,19 meses (Gráfico 3).
No teste Kruskal-Wallis, não houve significância estatística com relação aos tratamentos e à expectativa de vida dos pacientes $(\mathrm{p}=0,185)$.

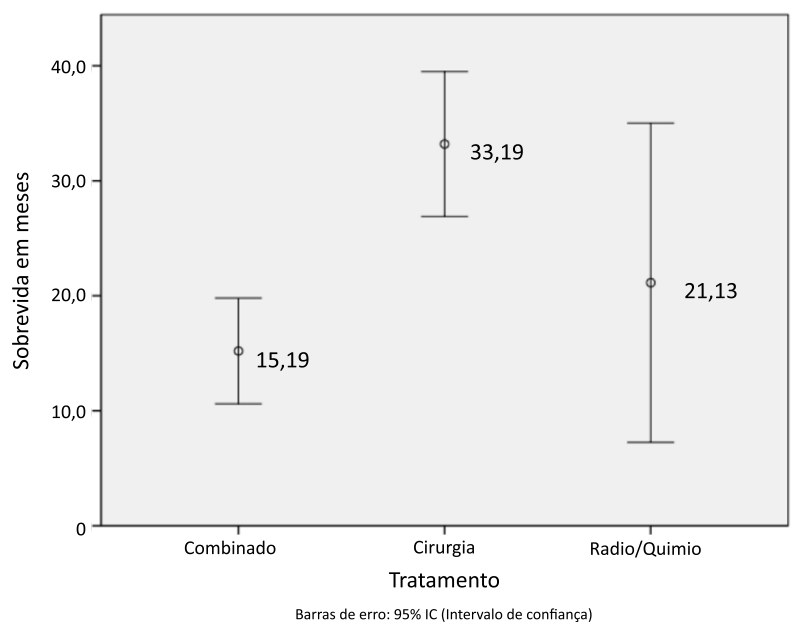

Gráfico 3. Intervalo e média de sobrevida dos pacientes que foram a óbito, segundo o tratamento recebido, período de 1993 a 2008. Passo Fundo, RS, Brasil

A média de sobrevida com relação ao local acometido pelo tumor (Gráfico 4) foi de 20,51 meses para a língua, 33,66 meses para o assoalho de boca e 27,92 meses para o palato. Pelo teste de Kruskal-Wallis, náo houve diferença estatística entre a sobrevida e a localização do tumor $(\mathrm{p}=0,201)$.

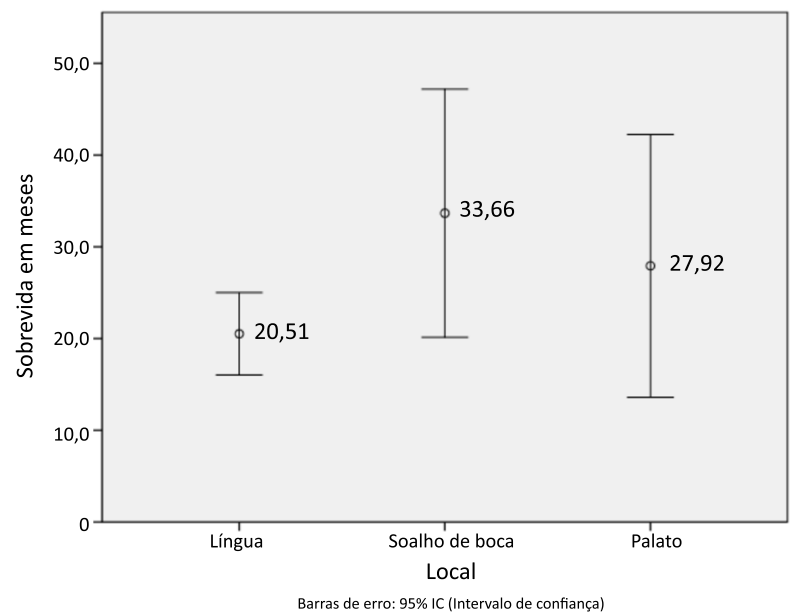

Gráfico 4. Intervalo e média da sobrevida dos pacientes que foram a óbito segundo a localização topográfica da lesão no diagnóstico, período de 1993 a 2008. Passo Fundo, RS, Brasil

\section{DISCUSSÃO}

O perfil do paciente acometido por carcinoma epidermoide oral em Passo Fundo e Regiáo mostra-se semelhante ao encontrado na literatura, sendo o sexo masculino, sexta década de vida e os leucodermas os mais comumente afetados. Outros estudos também 
identificaram dados semelhantes, sempre com o gênero masculino como o mais afetado e médias de idade entre a quinta e a sétima décadas de vida ${ }^{1,3,8}$.

O uso de álcool e tabaco apresentaram-se como os dois maiores fatores de risco para o desenvolvimento do carcinoma epidermoide oral. Quando associados, os fatores de risco são mais prevalentes do que isoladamente ${ }^{1,2,4,8,9}$. Neste estudo, tal associação chega a 84,7\%. O Estado do Rio Grande do Sul é responsável por $51 \%$ de toda a produçáo de tabaco do país e tem a quinta maior prevalência de câncer de boca do Brasil. A Organização Mundial da Saúde afirma que um terço de toda a população mundial é fumante. Segundo o Instituto Brasileiro de Geografia e Estatística $(\mathrm{IBGE})^{10}$, aproximadamente $25 \%$ de pessoas acima de 15 anos relatam esse hábito diário no Brasil. Mesmo com as campanhas de proteção ao indivíduo quanto ao uso do tabaco, ainda é grande o número de pessoas que faz esse uso ${ }^{10}$.

A média de sobrevida global dos 208 pacientes que foram a óbito em decorrência do câncer foi de 25,76 meses. A literatura aponta dados que mostram a diminuição na taxa de mortalidade de 2011 para $2015 \mathrm{em} \mathrm{3,8 \%} \mathrm{ao} \mathrm{ano} \mathrm{para}$ homens e 2,3\% ao ano para mulheres ${ }^{11}$. Essa sobrevida está relacionada inclusive a fatores geográficos, comportamentais e demográficos ${ }^{6}$. Outros estudos afirmam que a sobrevida dos pacientes acometidos por essa patologia diminui gradativa e proporcionalmente à medida que a severidade de doença avança; ou seja, quanto maior a severidade, maior o estádio, menor a sobrevida ${ }^{6,8,12}$.

O sítio anatômico mais acometido foi a língua, assim como mostrado em outros estudos, com maior ênfase para a base de língua ${ }^{1,8,13}$. Embora não tenha sido estatisticamente significante, pacientes acometidos por essa neoplasia na língua tiveram a pior sobrevida do que os demais locais, com 20,5 meses. A localização topográfica da lesão não mostrou influência estatística na sobrevida neste estudo, dados semelhantes também já foram identificados em estudos prévios ${ }^{8,14}$.

Embora a cavidade oral seja uma região anatômica de fácil acesso e visualização, muitas lesôes ficam despercebidas, atrasando o diagnóstico e piorando consequentemente o prognóstico ${ }^{11,12}$.

A detecção de lesóes potencialmente malignas pelo profissional como a leucoplasia e a eritroplasia contribui significativamente para o diagnóstico precoce do câncer bucal e deve ser analisada juntamente com o histórico médico do paciente, a presença ou não de hábitos deletérios, o envolvimento de linfonodos e análise de imagens radiográficas ou tomográficas ${ }^{15,16}$. As lesôes em estágio inicial, muitas vezes, apresentam-se sem sintomatologia dolorosa, o que pode não ser percebido pelo paciente, levando, por essa razão, frequentemente à demora no diagnóstico, assim como não há indicação na literatura para a estimulação da realização do autoexame, pelo fato de o paciente náo possuir um parâmetro que indique a condição de normalidade das mucosas ${ }^{16}$.

A implantaçáo de programas de saúde que visam a trabalhar na detecção precoce do câncer bucal por meio de uma triagem clínica tem sido discutida na literatura e se mostrado factível por apresentar a possibilidade da identificação de lesôes potencialmente malignas em exames simples; no entanto, a literatura náo apresentou evidências suficientes que cativassem os governos a investirem em verbas para tal realização ${ }^{16}$.

O diagnóstico tardio foi observado na maioria dos pacientes deste estudo, uma vez que $77,2 \%$ de todos os casos eram estádios III e IV, demonstrando, dessa forma, a demora na identificação e no início do tratamento dessa patologia. Ao observar os pacientes que foram diagnosticados em estágios iniciais da doença, verificou-se uma quantidade maior de pessoas vivas, e a sobrevida dos que foram a óbito foi muito superior à dos diagnosticados em estágios mais tardios. Os dados obtidos revelaram 2,5 vezes mais chance de um paciente ir a óbito do que quando o diagnóstico foi realizado precocemente. $\mathrm{O}$ estadiamento do tumor no momento do diagnóstico mostra-se um fator importante em relaçáo à sobrevida dos pacientes, pois a literatura revela que a diminuiçáo do tempo de vida do paciente diagnosticado em estágio tardio é muito inferior quando comparado ao estágio inicial ${ }^{1,6,9}$.

Este estudo revela que pacientes com diagnóstico tardio viveram em média 17,8 meses, enquanto os que foram diagnosticados em estágio inicial tiveram 46,2 meses de sobrevida. Apesar de a cavidade oral ser sítio de fácil acesso para o exame visual, nem sempre isso ocorre, por isso é fundamental que os pacientes mantenham consultas anuais para que os profissionais façam uma avaliaçáo da cavidade bucal como um todo, a fim de que pequenas lesóes possam ser diagnosticadas e tratadas precocemente.

O tratamento está intimamente relacionado ao tamanho do tumor, ao seu estadiamento e ao local acometido, tornando-se mais complexo quanto mais avançado o grau de invasão e a complexidade do sítio anatômico envolvido ${ }^{6,17}$. O tratamento de eleição encontrado nesta pesquisa, na maioria dos casos, foi o cirúrgico $(55,9 \%)$, que objetiva a remoção das células afetadas e uma maior possibilidade de eliminação do tumor. Os pacientes que receberam esse tipo de terapia tiveram sobrevida de 33,1 meses, a maior entre os tipos de tratamento analisados. $\mathrm{O}$ uso de terapias combinadas foi a escolha em pacientes com diagnóstico mais tardio, estando em consenso com a literatura encontrada ${ }^{1,3,6,17}$. 
O estágio clínico da doença é um dos fatores que mais influenciam o desenvolvimento de metástases de carcinoma de boca e orofaringe e isto também contribui para a escolha de tratamento combinado, o que é geralmente utilizado em lesōes mais avançadas ${ }^{3,12}$.

Apesar de existirem avanços imensuráveis nas últimas décadas no que se refere à tecnologia do tratamento para o câncer, a sobrevida dos pacientes depende muito mais do estadiamento da lesão do que do tratamento utilizado $^{1,3,6,12,17}$, o que foi também observado nesta pesquisa. O prognóstico dessa patologia pode se delinear a partir do estadiamento em que ela se encontra no momento do diagnóstico, tanto do ponto de vista clínico como de saúde pública ${ }^{3,4}$.

Diante do fato de muitas informaçôes de relevância clínica e epidemiológica estarem faltando nos prontuários analisados, se registra um alerta para os profissionais sobre a importância de reportarem tais informaçóes com a devida precisão, pois somente assim esse conhecimento permitirá açôes mais efetivas de prevenção e combate ao carcinoma epidermoide oral.

Em virtude da grande quantidade de pacientes que não tiveram seguimento (203 dos 327 contidos no banco de dados), sugere-se um descaso com os registros médicos, com várias hipóteses para essa situação, como por exemplo, falta de tempo, pessoal ou mesmo interesse por parte dos profissionais, o que realmente influencia diretamente os resultados desta e de outras pesquisas que possam depender dessas informaçóes. Outro ponto a ser discutido é se o investimento nesses tratamentos tem sido suficiente no combate a essa neoplasia; ou seja, é necessário reforçar um maior número de informaçôes nos bancos de dados para que essa avaliação seja precisa.

O Estado do Rio Grande do Sul tem o quinto maior índice de desenvolvimento humano do Brasil, 0,832, o que representa o nível de saúde, escolaridade e renda da população. O índice de Gini, que demonstra a concentração de renda da população, se apresenta na média $(0,500)^{10}$, sugerindo uma boa qualidade de vida dessas pessoas, o que deveria repercutir em uma maior informação e consequentemente no acesso aos serviços de saúde, diagnóstico e tratamento.

Uma análise histórica da situação do câncer em diversos países tem demonstrado que as açóes de controle da doença sempre foram eminentemente curativas em detrimento das preventivas e de diagnóstico precoce ${ }^{4-7}$. Estas definitivamente não representam uma boa estratégia no combate a essa patologia, considerando inclusive que os gastos utilizados para tratamento dos pacientes e dos que estatisticamente acabam evoluindo para o óbito são muito superiores para o governo do que os custos aplicados em medidas de prevenção.
Outro ponto é o fato de que países desenvolvidos e com maior índice de escolaridade da população tendem a apresentar taxas menores de câncer de boca na população de mais idade como a encontrada no presente estudo, no entanto apresentam uma crescente para os pacientes jovens, em maior tendência para mulheres leucodermas ${ }^{13}$.

Deve-se lembrar de que o estudo apresenta uma limitação de acesso aos dados de forma mais ampla pelo fato de estar restrito a um único hospital e ter assim o seu número reduzido, não podendo ampliar sua análise para o que ocorre no Estado e náo representando o reflexo de um país inteiro.

\section{CONCLUSÃO}

O HSVP apresentou um perfil de pacientes diagnosticados com carcinoma epidermoide oral predominantemente do sexo masculino, na sexta década de vida, leucoderma, com baixo grau de instruçáo e com hábito de tabagismo e etilismo associados e que foram, na sua maioria, diagnosticados com carcinoma epidermoide oral em estádios III e IV, e isto aumentou em 2,5 vezes a chance de evoluir para óbito. A cirurgia foi o tratamento de eleição na maioria dos casos, proporcionando assim uma maior sobrevida do paciente, embora não tenha mostrado significância estatística em relação aos demais tipos de tratamento. Esses dados contribuem para reforçar a proposta de incentivo para ampliar os investimentos em medidas de prevençâo, independentemente dos gastos públicos necessários às terapias de cura.

\section{CONTRIBUIÇÕES}

Luana Soares Kuze e Gisele Rovani contribuíram substancialmente na concepção e planejamento do estudo; na obtenção, análise e/ou interpretação dos dados. Adriano Pasqualotti, Ferdinando de Conto, Mateus Ericson Flores e Thales Henrique Jincziwski Ponciano contribuíram substancialmente na redação e revisão crítica. Todos os autores aprovaram a versão final a ser publicada.

\section{DECLARAÇÃO DE CONFLITO DE INTERESSES}

Nada a declarar.

\section{FONTES DE FINANCIAMENTO}

Não há.

\section{REFERÊNCIAS}

1. Tavares C, Guimarães J, Lopes O, et al. Epidemiological profile of malignant oral cancers in a population of 
northern Portugal. Rev Port Estomatol Cir Maxilofac. 2016;57(4):229-35. doi: https://doi.org/10.1016/j. rpemd.2016.10.145

2. Gupta B, Bray F, Kumar N, et al. Associations between oral hygiene habits, diet, tobacco and alcohol and risk of oral cancer: a case-control study from India. Cancer Epidemiol. 2017;51:7-14. doi: https://doi.org/10.1016/j. canep.2017.09.003

3. Zhang LW, Li J, Cong X, et al. Incidence and mortality trends in oral and oropharyngeal cancers in China, 20052013. Cancer Epidemiol. 2018;57:120-6. doi: https:// doi.org/10.1016/j.canep.2018.10.014

4. Petersen PE. Oral cancer prevention and control - The approach of the World Health Organization. Oral Oncol. 2009;45(4-5):454-60. doi: https://doi.org/10.1016/j. oraloncology.2008.05.023

5. Azevedo e Silva G, Moura L, Curado MP, et al. The fraction of cancer attributable to ways of life, infections, occupation, and environmental agents in Brazil in 2020. PLoS ONE. 2016;11(2):e0148761. doi: https://doi. org/10.1371/journal.pone.0148761

6. D'Cruz AK, Vaish R, Dhar H. Oral cancers: current status. Oral Oncol. 2018;87:64-9. doi: https://doi. org/10.1016/j.oraloncology.2018.10.013

7. Pearce A, Sharp L, Hanly P, et al. Productivity losses due to premature mortality from cancer in Brazil, Russia, India, China, and South Africa (BRICS): a populationbased comparison. Cancer Epidemiol. 2018;53:27-34. doi: https://doi.org/10.1016/j.canep.2017.12.013

8. Honorato J, Rebelo MS, Dias FL, et al. Gender differences in prognostic factors for oral cancer. Int J Oral Maxillofac Surg. 2015;44(10):1205-11. doi: https://doi. org/10.1016/j.ijom.2015.04.015

9. Wang W, Han S, Yao Z, et al. A study of epidemiologic and recurrence factors of oral cancer. J Oral Maxillofac Surg. 2012;70(9):2205-10. doi: https://doi.org/10.1016/j. joms.2011.09.040

10. Instituto Brasileiro de Geografia e Estatística. Pesquisa nacional por amostra de domicílios [Internet]. Vol. 30. Rio de Janeiro: IBGE; 2009 [acesso 2018 abr 12]. Disponível em: chrome-extension:// efaidnbmnnnibpcajpcglclefindmkaj/viewer.html?pdfu $\mathrm{rl}=\mathrm{https} \% 3 \mathrm{~A} \% 2 \mathrm{~F} \% 2 \mathrm{Fbiblioteca}$. ibge.gov.br\%2Fvisu alizacao\%2Fperiodicos\%2F59\%2Fpnad_2009_v30_ br.pdf\&chunk=true

11. Instituto Nacional de Câncer José Alencar Gomes da Silva [Internet]. Rio de Janeiro: INCA; [data desconhecida]. Observatório da Política Nacional de Controle do Tabaco: mortalidade no Brasil; [modificado 2021 maio 20; acesso 2021 nov 17]. Disponível em: https:// www.inca.gov.br/observatorio-da-politica-nacional-decontrole-do-tabaco/mortalidade-brasil
12. D'souza S, Addepalli V. Preventive measures in oral cancer: an overview. Biomed Pharmacother. 2018;107:72-80. doi: https://doi.org/10.1016/j.biopha.2018.07.114

13. Cohen Goldemberg D, Araújo LHL, Antunes HS, et al. Tongue cancer epidemiology in Brazil: incidence, morbidity and mortality. Head Neck. 2018;40(8):183444. doi: https://doi.org/10.1002/hed.25166

14. Kademani D, Bell RB, Bagheri S, et al. Prognostic factors in intraoral squamous cell carcinoma: the influence of histologic grade. J Oral Maxillofac Surg. 2005;63(11):1599-605. doi: https://doi.org/10.1016/j. joms.2005.07.011

15. Scully C, Sciubba JJ, Bagan JV. Oral mucosal precancer and cancer: a helpful discriminating clinical tool. Med Oral Patol Oral Cir Bucal. 2015;20(5):e587-e90. doi: https://doi.org/10.4317/medoral.20155

16. Speight PM, Epstein J, Kujan O, et al. Screening for oral cancer-a perspective from the Global Oral Cancer Forum. Oral Surg Oral Med Oral Pathol Oral Radiol. 2017;123(6):680-7. doi: https://doi.org/10.1016/j. oooo.2016.08.021

17. Shanti RM, O’Malley Jr BW. Surgical management of oral cancer. Dent Clin North Am. 2018;62(1):77-86. doi: https://doi.org/10.1016/j.cden.2017.08.005

Recebido em 22/12/2020 Aprovado em 13/4/2021 